\title{
Improving data interpretation from SHTB tests on ductile metals
}

\author{
Avishay Lindenfeld*, and Yehuda Partom \\ RAFAEL, P.O. Box 2250, Haifa 3102102, ISRAEL
}

\begin{abstract}
This work presents a methodology to calibrate a strength model for ductile metals, based on dynamic tension tests of relatively long Dog-Bone specimens conducted on a Split Hopkinson Tension Bar (SHTB). We address the main difficulties involved in conducting and interpreting such tests, namely the duration of the loading pulse needed to deform long specimens and the non-uniform stress and strain distributions along the specimen due to neck formation.

The first issue is addressed by using the waves' reflections within the output bar, as explained below. When the first loading (tension) wave does not cause failure of the specimen, a reflected compression wave travels from the specimen's bar end to the free bar's end. Upon reaching the free end this latter compression wave is reflected again as a second tension wave, which travels back along the bar until it reaches the specimen and loads it the second time. This enables further deformation of the specimen, practically doubling the loading pulse duration without changing the striker's length.

The second issue is addresses by using full numerical simulations of the experimental setup, including the striker, the bars and the specimen. This way, the full dynamic behaviour of the specimen is taken into account, eliminating the need to consider specimen equilibrium and taking into account the current strain rate in the specimen as it deforms. Hence, model calibration can be done from the very start of plastic deformation and without the need to keep the strain rate constant during deformation. As a result, it is possible to reliably calibrate the strength model considering necking and neck location, as well as plastic heating which is a significant factor in the plastic deformation of ductile metals.
\end{abstract}

\section{Introduction}

The Split Hopkinson Tension Bar (SHTB) is a wellknown technique to characterize plastic flow and failure in uniaxial tension $[1,2]$. With these modifications to the classical Split Hopkinson Pressure Bar (SHPB) [3], applying the classical analysis may become questionable, as the onset of necking creates a nonuniform distribution of stress and strain [4, 5]. Various techniques have been suggested to overcome this difficulty.

Rajendran and Bless [6] use Bridgman's approximation [7] to obtain a stress-strain curve in dynamic tension beyond the point of necking. To this end, they measure the geometry of the neck, using high speed photography. They find this approach to have different levels of accuracy for different materials. Rodriguez et al. [8], using finite element computations, suggest using an 'effective length' as a representative length, for which uniform strain can be assumed in a specimen, thus enabling the use of the classical analysis. However, they determine this effective length using numerical simulations. Rotbaum et al. [9] suggest using long specimens, to keep the stress and strain distribution constant in a large part of the specimen prior to necking. In this way they are able to use the classical analysis with minimal error, but only for plastic strains of up to $3 \%$. Mirone [10] uses a correction to the classical analysis, which enables reconstruction of the stressstrain curve until failure, but within an error of $15-25 \%$.
Here we use numerical simulations of the entire experimental setup to predict the specimen stress-strain response until failure, including details of the neck formation. We change our strength model parameters and repeat the simulation until we match the velocity histories measured in the bars.

Another technical difficulty in these tests is related to the relatively large elongation needed to cause failure in ductile specimens. For a given SHTB setup, the energy available for loading a specimen depends on the pulse duration and amplitude, which are dictated by the striker's length and velocity, respectively. A way to achieve a long pulse is by using a long striker [11], but this may not be feasible for an existing lab setup. Instead, we use a technique based on wave reflections in the input and output bars, practically doubling the loading pulse duration for an existing SHTB setup. Combining this technique with a numerical simulation of the entire experimental setup, it is possible to directly interpret the recorded pulses in the bars without regard to specimen equilibrium.

In section 2 we describe the experimental setup of our SHTB setup using circular Dog-Bone (DB) specimens, and present the technique that enables to practically double the duration of the loading pulse. In section 3 we describe our computational scheme and in section 4 we present the calibration method of a strength model in both compression and tension.

*Corresponding author: avishay.lindenfeld@gmail.com 


\section{Experimental procedure and setup}

As a representative material we use Aluminum 6061T651, which fails in tension at total (average) strain of above $20 \%$. First, we calibrate our strength model for this material in compression, using a SHPB. Then, assuming that the strength model parameters hold approximately in tension as well, we conduct simulations to determine the setup for the tension tests. At the end we verify the validity of this assumption from the tension tests.

\subsection{Compression tests}

We use our standard SHPB setup, made of 25.4 diameter hardened C300 maraging steel bars. The striker, input and output bar lengths are 40, 300 and $250 \mathrm{~cm}$, respectively, see Fig. 1a. In both compression and tension we use our laser interferometry-based measurement system [12], to recoded particle velocity histories of the input and output bar surfaces at points around $1 \mathrm{~m}$ from the specimen. We use disk shaped specimens with $\mathrm{L}_{0} / \mathrm{D}_{0}=0.5 \mathrm{~mm}$ [13] and with diameters of 10,12 and $14 \mathrm{~mm}$ and appropriate striker velocities are able to cover strain rates in the range of $600 \div 4000$ $\sec ^{-1}$.

a)

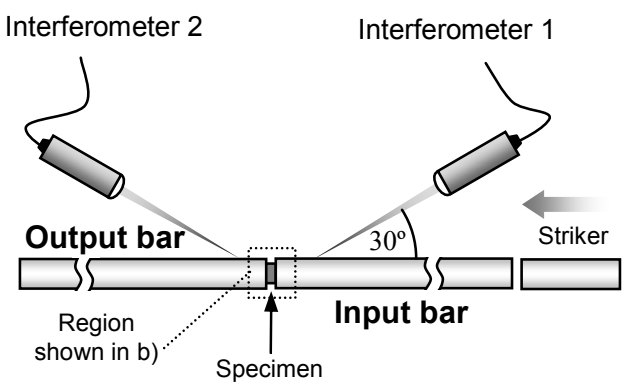

b)

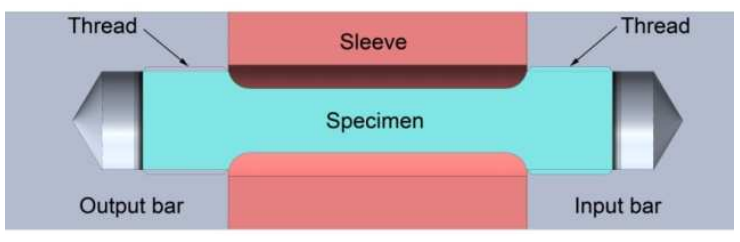

Fig. 1. Experimental setup of the interferometry-based SHB: a) compression b) tension (cut view).

\subsection{Tension tests}

For the tension tests we use aluminum 7075 bars (with the same diameter as before), to reduce the impedance mismatch between the bars and the specimens. The striker and the input and output bar lengths are 75, 300 and $220 \mathrm{~cm}$, respectively. Tension of the specimen is achieved by the reflected compression wave in the output bar from its far boundary [1]. This tension wave then loads the specimen through the threads shown in Fig. 1b.

Our preliminary simulations showed that for some specimens, the duration of the tension wave generated by the above-mentioned striker might not be long enough to cause failure. To prolong the loading, we use the second reflection in the output bar as an additional loading wave, see Fig. 2. In this way, the loading duration of the specimen is practically doubled. Even though the second wave is weaker than the first one due to loss of energy to deform the specimen, it is still strong enough to cause failure in the specimen. Comparing the specimen response between one long loading wave and two shorter successive waves as presented here, our simulations show that there is no difference in the strain distribution or in the neck location along the specimen. Hence, this is a valid method to characterize the material response in SHTB tests.

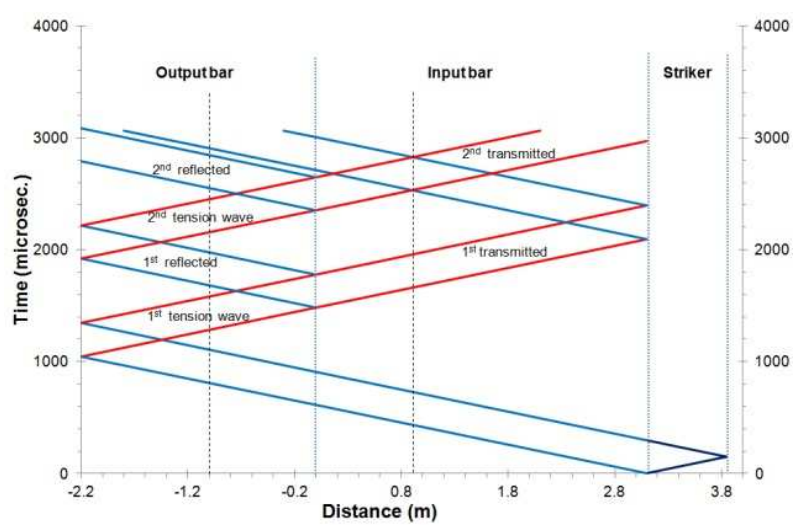

Fig. 2. $x$-t diagram of the SHTB experiment with two loading waves; blue - compression, red - tension.

Taking into account our SHTB system diameter and our maximum striker length, a set of tests was designed so that some specimens would not fail, to better calibrate the strength model. This was achieved by choosing specimen diameter of $7.5 \mathrm{~mm}$ and gauge lengths of 22.5 , 30 and $37.5 \mathrm{~mm}$, so that their $\mathrm{L}_{0} / \mathrm{D}_{0}$ are 3,4 and 5 , respectively. The specimen geometry and dimensions are shown in Fig. 3. In the current work we only address the strength model calibration, where the failure reproduction and calibration will be addressed in a future work.

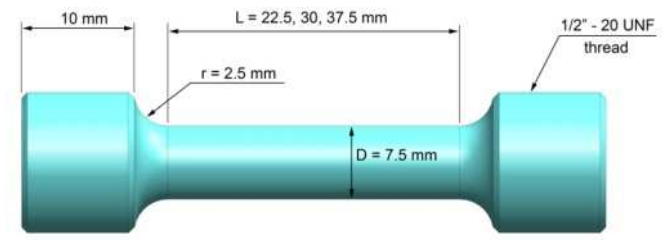

Fig. 3. Geometry and dimensions of the SHTB specimens.

\section{Computer simulations}

Using numerical simulations of the entire SHB system to interpret test results has the advantage of eliminating the inaccuracies induced in the tests, as explained below. In the classical SHPB equations, one ignores the low strain part because of the initial lack of equilibrium in the specimen. However, by comparing the recorded signals 
with the computed ones, it is possible to obtain the material stress-strain curve from the very beginning of plastic deformation, avoiding the need to disregard the low plastic strain regime. Another advantage of our numerical calibration approach, as will be shown in section 4 , is the ability to easily take into account the effect of thermal softening, since considering TS within the framework of the classical analysis is not straightforward. Thus, to accurately calibrate the material response we rely on numerical simulations for both compression and tension, and use the classical analysis only as a reference.

We use an in-house 2D code, which was previously used in other work [14]. This code uses a finite difference explicit scheme, where J2 plasticity is implemented in the scheme following Wilkins' radial return method for elastic-viscoplastic flow [15]. To represent elastic-viscoplastic response we use the Johnson-Cook (JC) strength model [16]:

$$
Y=\left(A+B \varepsilon_{p}^{n}\right) \cdot\left(1+C \ln \frac{\dot{\xi}_{p}}{\varepsilon_{n}}\right) \cdot\left[1-\left(\frac{T-T_{0}}{T_{m m}-T_{n}}\right)^{m}\right]
$$

where $A, B, n, C$ and $\mathrm{m}$ are material parameters, $\dot{\varepsilon}_{0}$ is a reference strain rate, taken to be $\varepsilon_{0}=1 \mathrm{sec}^{-1}, \mathrm{~T}_{0}$ is the initial temperature (room temperature here) and $T_{m}$ is the melting temperature. All the simulations use resolution of 10 elements $/ \mathrm{mm}$ in the specimen, which was found to be convergent.

\section{Experimental results and model calibration}

\subsection{Compression tests}

The raw signals from one of the tests $\left(D_{0}=14 \mathrm{~mm}\right.$, $\mathrm{V}_{\text {striker }}=30 \mathrm{~m} / \mathrm{sec}$ ) is presented in Fig. $4 \mathrm{a}$, together with the numerically computed signals that include TS. The computed results are shown after calibration of the JC model parameters, emphasizing the reproduction of the transmitted pulse since it has less noise. The calibrated parameters of the JC model are shown in Table 1, with $\mathrm{T}_{\mathrm{m}}=900{ }^{\circ} \mathrm{K}$ and $\mathrm{m}=1$ as representative values. As shown in Fig. 4b (red line), very good agreement is achieved between the experimental and the numerical curves for the simulation which include TS. The same level of agreement is obtained for all other tests (not shown here).

Table 1. Material parameters for the JC model, calibrated from compression tests.

\begin{tabular}{cccccc}
\hline $\begin{array}{c}A \\
(\mathrm{MPa})\end{array}$ & $\begin{array}{c}B \\
(\mathrm{MPa})\end{array}$ & $C$ & $n$ & $\begin{array}{c}T_{m} \\
\left({ }^{\circ} \mathrm{K}\right)\end{array}$ & $m$ \\
\hline 339 & 180 & 0.015 & 0.36 & 900 & 1 \\
\hline
\end{tabular}

Another advantage of the above calibration method is the ability to easily include the effect of TS. To demonstrate the importance of TS, we calibrated another set of parameters for the JC model, this time without taking into account TS. The best agreement we achieved with the experimental results is shown in Fig. $4 \mathrm{~b}$ (green line), for the parameters $A=290 \mathrm{MPa}, B=140 \mathrm{MPa}, C$ $=0.015$ and $n=0.15$. As can be seen, the computed curve without TS has a higher deviation from the experimental signal compared to the computed curve with TS, expressed by a higher slope (higher strain hardening) of the calibrated curve without TS relative to the experimental signal.

a)
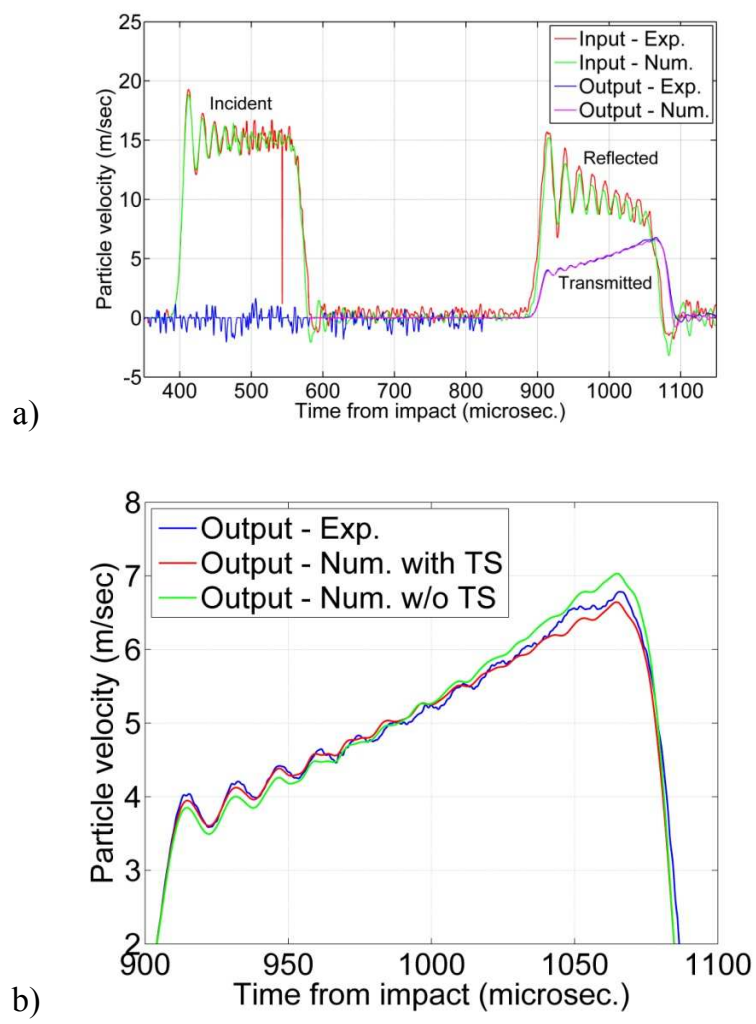

Fig. 4. Compression tests - comparison between experimental and numerical results: a) all signals b) transmitted signal.

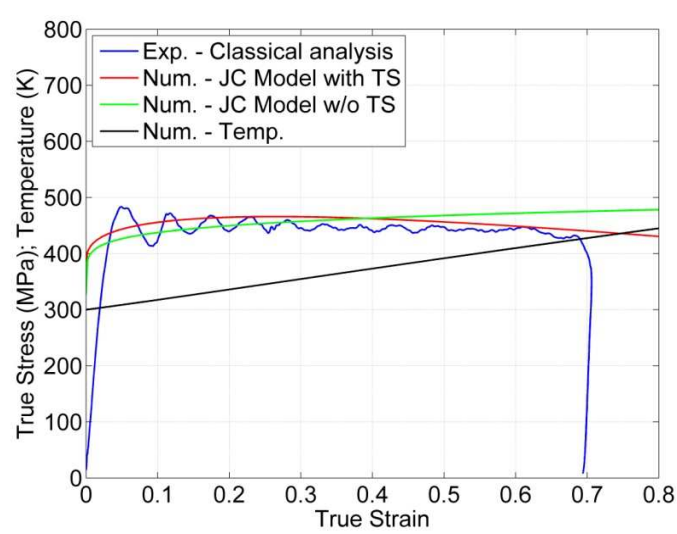

Fig. 5. Comparison of the stress-strain curve from the classical analysis to the numerically calibrated curves, for the same test shown in Fig. 4.

Comparison of the stress-strain curve from the standard (classical) analysis [3] to those from the numerical analysis is presented in Fig. 5. There is a general agreement between the classical analysis and the 
calibrated JC model with TS, although, as expected, for plastic strain up to $5 \%$ there is a significant difference between the curves. The eventual decrease in the stress level of the model which includes TS for higher strains is a result of the temperature rise in the specimen during plastic deformation, which is about $100^{\circ}$ for plastic strain of 0.6, as seen in Fig. 5.

\subsection{Tension tests}

The recorded signals from the test on the 'medium length' specimen which did not fail $\left(\mathrm{L}_{0} / \mathrm{D}_{0}=4, \mathrm{~V}_{\text {striker }}=15\right.$ $\mathrm{m} / \mathrm{sec}, \bar{\varepsilon}_{\text {average }} \approx 340 \mathrm{sec}^{-1}$ ) are shown in Fig. 6. One should note that the loading conditions (stress, strain rate, etc.) for the second wave are different from the first wave, since part of the energy is dissipated within the specimen during the first loading wave and since the specimen elongates before the second loading. However, these effects are inherently taken into account by the simulations and hence do not need special consideration.

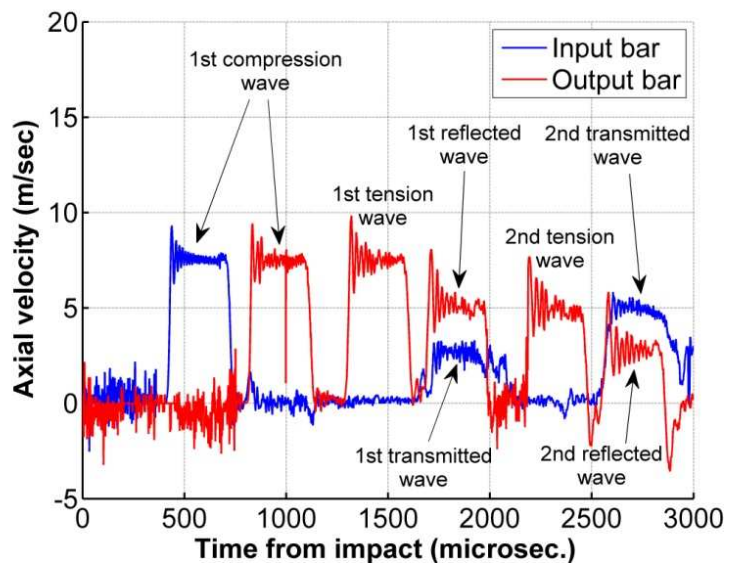

Fig. 6. Recorded signals for SHTB test with specimen $\mathrm{L}_{0} / \mathrm{D}_{0}=4$, $\mathrm{V}_{\text {striker }}=15 \mathrm{~m} / \mathrm{sec}$.

Comparison between the experimental and the computed signals, based on the calibrated model given in Table 1 is shown in Fig. 7, and the agreement is very good. Similar agreement was obtained for the other tests. An example of the computed neck evolution in a twowave loading structure is shown in Fig. 8.

Another parameter which we use to validate the numerical results is the elongation of the gauge length. This parameter represents the integral behaviour of the specimen during the whole deformation process, including necking, and hence is another reliable measure for validation. The comparison between the measured and the computed specimen elongation was found to be within $9 \%$, which is satisfactory considering measurement error which is less than $2 \%$. Hence, we conclude that for Al606-T651, there is no significant difference between the strength model parameters in uniaxial tension and compression.

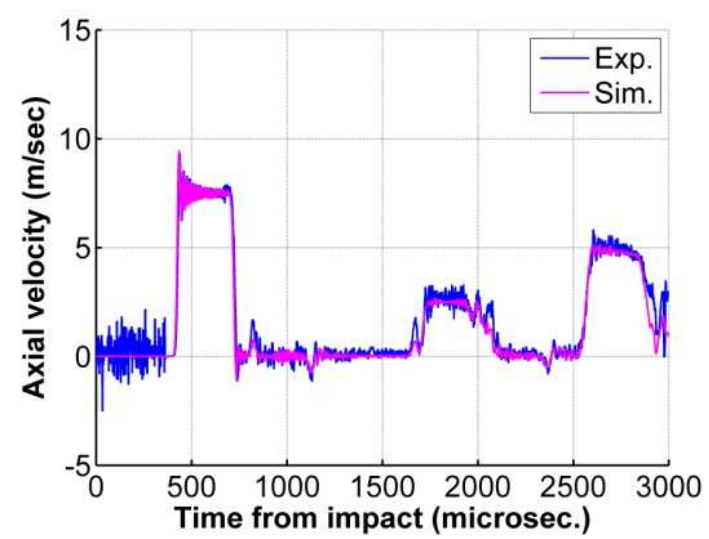

b)

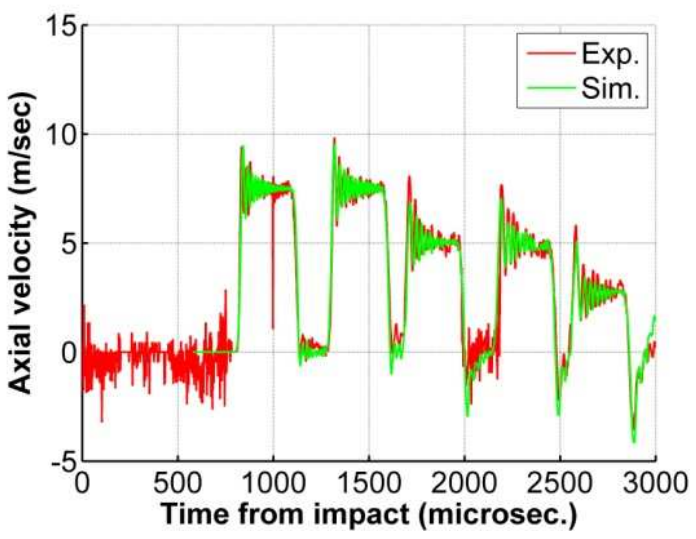

Fig. 7. Comparison between the recorded and the computed signals for SHTB test with specimen $\mathrm{L}_{0} / \mathrm{D}_{0}=4, \mathrm{~V}_{\text {striker }}=15$ $\mathrm{m} / \mathrm{sec}$ : a) Input bar, b) Output bar.

\section{Summary and conclusions}

We focus here on the response of ductile metals to uniaxial tensile dynamic loading via the SHTB system. We use a combined experimental and numerical approach with Al6061-T651 as a model material, circular dog-bone specimens with gauge length to diameter ratios of 3,4 , and 5 and the JC constitutive model.

Based on direct comparison of the velocity signals measured in the tests to the ones predicted by simulations of the full experimental setup, we present a calibration method that has several advantages over the standard calibration based on the classical analysis:

- Accurate prediction of the stress-strain curve, in the low strain region for compression tests and for the full strain range for tension tests. This enables to ignore equilibrium considerations in the specimen and to take into account neck formation.

- Inherent and simple calibration of thermal softening, which is not straightforward with the standard analysis.

- The ability to cause failure in relatively long specimens, by using more than one tensile loading wave in the tension tests. We are not aware that this method has been used before. 


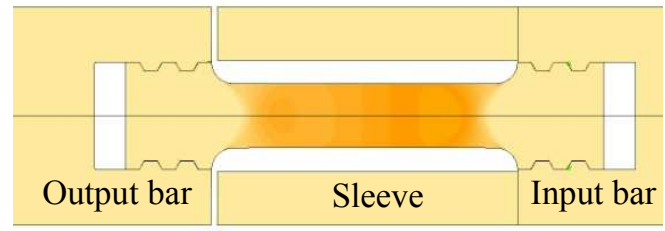

a) $\mathrm{t}=1540 \mu \mathrm{sec}$

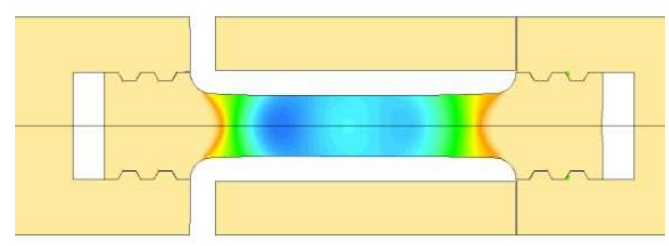

c) $\mathrm{t}=1700 \mu \mathrm{sec}$

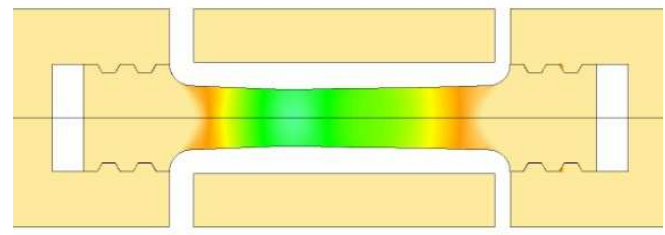

e) $\mathrm{t}=2420 \mu \mathrm{sec}$

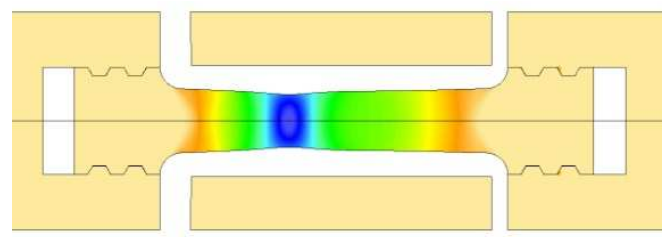

g) $\mathrm{t}=2500 \mu \mathrm{sec}$

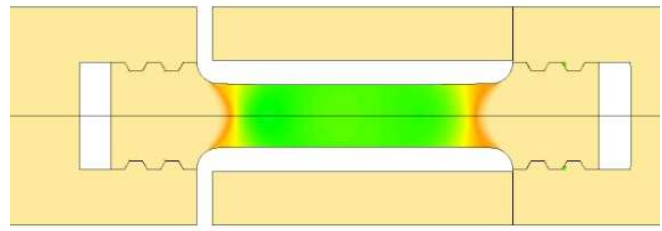

b) $\mathrm{t}=1620 \mu \mathrm{sec}$

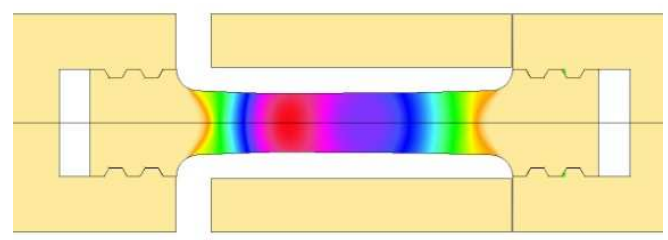

d) $\mathrm{t}=1780 \mu \mathrm{sec}$
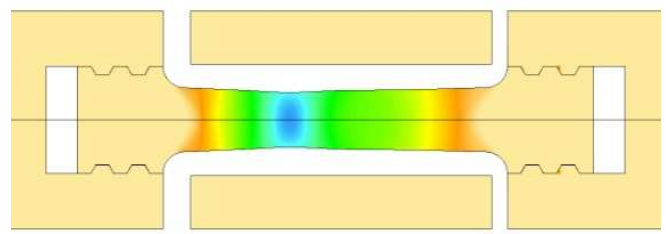

f) $\mathrm{t}=2460 \mu \mathrm{sec}$

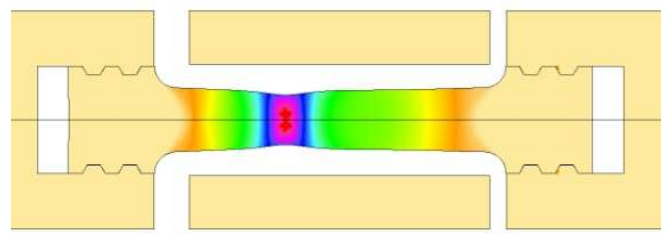

h) $\mathrm{t}=2540 \mu \mathrm{sec}$

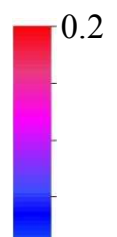

0 0.6

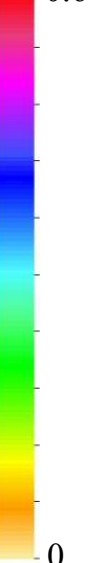

Fig. 8. Computed time evolution of the neck for specimen $L_{0} / D_{0}=4, V_{\text {striker }}=19.5 \mathrm{~m} / \mathrm{sec}$, effective plastic strain color maps; a)-d) first tension wave, e)-h) second tension wave.

\section{References}

1. T. Nicholas, Exp. Mech. 62:177-185 (1981)

2. G.H. Staab, A. Gilat, Exp. Mech. 313:232-235 (1991)

3. H. Kolsky, Proc. Phys. Soc. B 62:676-700 (1949)

4. A. Gilat, T.E. Schmidt, A.L. Walker, Exp. Mech. 49:291-302 (2009)

5. G. Besnard, F. Hild, J.M. Lagrange, P. Martinuzzi, S. Roux, Int. J. Impact Eng. 49:179-191 (2012)

6. A.M. Rajendran S.J. Bless, Exp. Mech. 26:319-323 (1986)

7. P.W. Bridgman, Trans. ASM 32:553-574 (1994)

8. J. Rodríguez, C. Navarro, V. Sánchez-Gálvez, J. Test. Eval. 22:335-342 (1994)

9. Y. Rotbaum, D. Rittel, Exp. Mech. 54:1205-1214 (2014)

10. G. Mirone, Mech. Mater. 58:84-96 (2013)

11. R. Gerlach, C. Kettenbeil, N. Petrinic, Int. J. Impact Eng. 50:63-67 (2012)

12. C. Avinadav, Y. Ashuach, R. Kreif, Rev. Sci. Instrum. 82, 073908 (2011)

13. L.D. Bertholf, C.H. Carnes, J. Mech. Phys. Solids 23:1-19 (1949)
14. Z. Lovinger, Y. Ashuach, O. Firstenberg, Proc. $9^{\text {th }}$ Int. Conf. Mech. Phys. Behaviour Mater. Under Dynamic Loading, DYMAT (2009)

15. M.L. Wilkins, In: Calculation of elastic-plastic flow, In: Methods in computational physics, vol. 3, (Academic Press 1964)

16. G.R. Johnson, W.H. Cook, Proc $7^{\text {th }}$ Int Symp Ballist, (1983) 
